\title{
Herausforderungen für die Qualitätssicherung und Qualitätsentwicklung in der Langzeitpflege
}

\section{KLAUS WINGENFELD}

Klaus Wingenfeld, Soziologe (MA) Dr. Public Health, wissenschaftlicher Geschäftsführer des Instituts für Pflegewissenschaft an der Universität Bielefeld

In der fachlichen und sozialpolitischen Diskussion um die Qualitätssicherung in der pflegerischen Versorgung zeichnet sich seit einiger Zeit ein wachsendes Bedürfnis nach grundlegender Neuorientierung ab. Schon seit vielen Jahren werden bessere und verlässlichere Methoden der Qualitätsbeurteilung gefordert. Die bestehenden externen Prüfsysteme sehen sich zunehmend mit Skepsis und der Frage nach dem Nutzen des inzwischen sehr hohen Prüfaufwandes konfrontiert. Auch die Ausrichtung des Qualitätsmanagements der Einrichtungen wird mehr und mehr hinterfragt. Der vorliegende Beitrag bemüht sich um eine Standortbestimmung.

\section{Einführung}

Die Einführung der Pflegeversicherung Mitte der 1990er Jahre hat nicht nur neue Grundlagen für die strukturelle Absicherung der Langzeitpflege geschaffen, sondern auch zu einer erheblichen Aufwertung der Qualitätssicherung und Qualitätsentwicklung in der Pflege geführt. Mit den Medizinischen Diensten der Krankenversicherung (MDK) wurde neben den Heimaufsichtsbehörden eine zweite externe Prüfinstanz mit der Kontrolle und Bewertung der Versorgungsqualität in Pflegeeinrichtungen beauftragt. Außerdem schrieb der Gesetzgeber den Spitzenverbänden der Pflegekassen die Kompetenz zu, Qualitätsrichtlinien festzulegen, nach denen externe Prüfungen im Rahmen des SGB XI durchzuführen sind. Hierdurch entstanden Strukturen, die für die Qualitätssicherung im Bereich der
Langzeitpflege bis heute prägend sind.

Einen erheblichen Entwicklungsschub löste die Pflegeversicherung auch auf dem Feld des internen Qualitätsmanagements aus. Parallel zum neuen MDKPrüfsystem erwuchsen aus anfänglich sehr individuellen und wenig geregelten Ansätzen anspruchsvolle Konzepte. Vor allem große Einrichtungsträger und einige Träger- bzw. Fachverbände bemühten sich um neue Organisationsformen und Verfahren zur Sicherung der Versorgungsqualität. Diese Entwicklung verlief allerdings sehr ungleichmäßig, so dass das interne Qualitätsmanagement in den Pflegeeinrichtungen nach wie vor große Unterschiede aufweist.

Parallel zu diesen Prozessen setzte eine anhaltende Diskussion über geeignete Strategien der Qualitätssicherung und Qualitätsbeurteilung ein, die im Laufe der Jahre eher noch an Intensität gewonnen hat. Verschiedene Faktoren 
haben dazu beigetragen. Dazu gehört unter anderem die Berichterstattung in den Medien, die häufig darauf ausgerichtet ist, Mängel der pflegerischen Versorgung aufzudecken - tatsächliche und vermeintliche. Wiederholt wurden Missstände in einzelnen Einrichtungen zur globalen Gefährdung pflegebedürftiger Menschen in Altenheimen hochstilisiert, und wiederholt wurden auch die Ergebnisse von Qualitätsprüfungen zu passenden Schlagzeilen uminterpretiert. Das Bild einer grenzwertigen Pflege (»Bilanz des Grauens" - Bildzeitung v. 31.8.2007), das hierdurch und durch andere Entwicklungen mitunter entstanden ist, hat das Bedürfnis nach objektiven und unmissverständlichen Qualitätsbeurteilungen erheblich befördert.

Auch die Unzufriedenheit mit den Kriterien externer Qualitätsprüfungen trägt dazu bei, dass dieses Bedürfnis wächst. Schon seit vielen Jahren wird bemängelt, dass die Ergebnisqualität im Verhältnis zur Struktur- und Prozessqualität in der Pflege nicht ausreichend berücksichtigt werde. Insbesondere die

\section{Schon seit vielen Jahren wird bemängelt, dass die Ergebnisqualität im Verhältnis zur Struktur- und Prozessqualität in der Pflege nicht ausreichend berücksichtigt werde.}

aktuellen Formen der Beurteilung von Prozessqualität zieht Kritik auf sich. Die Fachlichkeit von Prozessen kann rückwirkend nur anhand von Aufzeichnungen beurteilt werden. Daher sind es vor allem die Qualitätshandbücher und die Pflegedokumentation, nach denen die Beurteilung erfolgt. Auf diese Weise entsteht bei den Mitarbeitern von Pflegeeinrichtungen und -diensten häufig der Eindruck, dass weniger die Unterstützung des Pflegebedürftigen als vielmehr die Qualität der schriftlichen Aufzeichnungen im Mittelpunkt der Bewertung steht. Lücken in der Dokumentation können zur Folge haben, dass bezweifelt wird, ob fachgerecht gepflegt wurde.

\section{Qualitätstransparenz}

Die mit dem Pflege-Weiterentwicklungsgesetz initiierten Neuerungen auf dem Feld der Qualitätsbeurteilung führten in verschiedenen Punkten zu einer Zuspitzung der Diskussion. Zum einen erhöhte sich mit der Reform die Prüfdichte. Pflegeeinrichtungen müssen sich nunmehr jährlich einer unangemeldeten Prüfung durch einen MDK oder vergleichbare Organisationen unterziehen. Zum anderen verpflichtete der Gesetzgeber die Landesverbände der Pflegekassen dazu, sicherzustellen, »dass die von Pflegeeinrichtungen erbrachten Leistungen und deren Qualität, insbesondere hinsichtlich der Ergebnis- und Lebensqualität, für die Pflegebedürftigen und ihre Angehörigen verständlich, übersichtlich und vergleichbar sowohl im Internet als auch in anderer geeigneter Form kostenfrei veröffentlicht werden « ( $\mathbb{S} 115$ Abs. 1a SGB XI). Ergebnisse externer Prüfungen, die so genannten Transparenzberichte, sind seither für jeden Interessierten im Internet einzusehen. Nach bestimmten Bewertungsregeln wird aus den Prüfergebnissen eine Note (analog zu Schulnoten) für die betreffende Einrichtung abgeleitet. Kriterien und Bewertungsregeln wurden, wie gesetzlich vorgegeben, durch den Spitzenverband Bund der Pflegekassen und die Repräsentanten der Leistungsanbieter, der überörtlichen Träger der Sozialhilfe und der kommunalen Spitzenverbände auf Bundesebene gemeinsam festgelegt bzw. vereinbart

(»Pflege-Transparenzvereinbarungen «).

Die Erwartungen an die öffentlichen Qualitätsberichte, die durch die erhöhte Prüfdichte und den damit verbundenen vermehrten Ressourcenverbrauch der Prüfinstitutionen besonders hoch gesteckt waren, blieben allerdings unerfüllt. Stattdessen entwickelte sich eine Kontroverse um mögliche Auswege aus den vielfältigen Problemen, die schon bald nach Umsetzung der neuen Prüfvorgaben sichtbar wurden.

$\mathrm{Zu}$ diesen Problemen gehört unter anderem die stetige Verbesserung der Prüfergebnisse mit der Folge einer Angleichung der Noten. Im Dezember 2011 ergab sich für ambulante Pflegedienste im Bundesdurchschnitt die Qualitätsnote 1,7. Stationäre Pflegeeinrichtungen erreichten mit einem Notendurchschnitt von 1,3 ein noch besseres Ergebnis. Ende 2011 gab es kein Bundesland mehr, in dem der Notendurchschnitt für die stationäre Pflege schlechter als 1,7 ausfiel (Newsletter Pflegenoten 2011). Vermutlich ist es nur eine Frage der Zeit, bis die Bewertungen fast aller Einrichtungen so nahe beim uneingeschränkten »sehr gut « liegen, dass Qualitätsunterschiede nicht mehr erkennbar sind und sich das neue Beurteilungssystem endgültig ad absurdum geführt hat.

Es bedarf kaum der Erläuterung, dass der anhaltende Trend zu immer besseren Noten nicht mit tatsächlichen Verbesserungen der Versorgung gleichgesetzt werden darf. Die Einrichtungen passen sich einfach an - genauer gesagt: Sie passen ihre Pflegedokumentation an. Sie nutzen gewissermaßen jene Schwachstelle im Prüfsystem, die so häufig Kritik auf sich gezogen hat, nämlich den Umstand, dass das Prüfergebnis in sehr hohem Maße von der Güte der Dokumentation abhängt.

Es gibt eine Reihe weiterer Probleme. Die Stichprobenkonstruktion beispielsweise führt dazu, dass häufig der Zufall darüber entscheidet, ob seltene Ereignisse oder Maßnahmen bei der Qualitätsbeurteilung überhaupt berücksichtigt werden können. Es kommt regelmäßig vor, dass sich die Beurteilung bestimmter Qualitätskriterien lediglich auf Informationen zur Pflegesituation eines einzigen Patienten bzw. Bewohners stützt. Außerdem zeigt die Bewertungssystematik Schwächen. Sie schließt die Gleichzeitigkeit eines »mangelhaft « bei wichtigen Einzelkriterien und eines »sehr gut « bei der Gesamtbewertung nicht aus. Überhaupt führt die Verwendung von Schulnoten zu Missverständnissen oder zumindest offenen Fragen. Was bedeutet die Note "gut « $(2,0)$ ? Nicht wenige Einrichtungen sind über ein solches Prüfergebnis enttäuscht, da der Landes- oder Bundesdurchschnitt inzwischen deutlich darunter liegt.

Darüber hinaus steht die Benotung mitunter im Gegensatz zu den bei der Prüfung tatsächlich festgestellten Mängeln. Dies jedenfalls war das Ergebnis einer Teilstudie, die von ausgewählten Medizinischen Diensten im Rahmen der Evaluation der Transparenzvereinbarungen durchgeführt wurde. Und nicht zuletzt ist darauf hinzuweisen, dass die "Transparenzkriterien « nicht der Intention des $\mathbb{} 115$ SGB XI genü- 
gen, insbesondere die Ergebnisqualität zu beurteilen. So etwa im Falle der stationären Versorgung: Abgesehen von einer Bewohnerbefragung zur Bewertung ausgewählter Aspekte der pflegerischen Versorgung finden sich unter mehr als 60 Einzelkriterien gerade einmal zwei, die man aus wissenschaftlicher Sicht als Kriterien der Ergebnisqualität bezeichnen kann.

Angesicht der guten Bewertungen erscheint es ein wenig paradox, dass viele Einrichtungen den »Pflegenoten « heute sehr skeptisch gegenüberstehen. Dazu beigetragen hat, dass der Aufwand, der zur Erreichung einer guten Beurteilung betrieben wird, hoch ist, dass viele Mitarbeiter sich und ihre Arbeit dennoch nicht in den Noten wiederfinden und dass die zentralen Fragen der internen Qualitätssicherung eben nicht auf der Ebene der Dokumentation lösbar sind.

Trotz der Ernüchterung, die sich inzwischen eingestellt hat, gibt es auf der Bundesebene manche Tendenzen, die vorliegenden Transparenzkriterien im Grundsatz beizubehalten und durch Nachbesserungen an Details die größten Probleme zu beheben. Aussichtsreich ist dieses Unterfangen allerdings nicht. Mit den vorliegenden Kriterien und den dazugehörigen Bewertungsgrundsätzen ist eine methodisch überzeugende Lösung kaum vorstellbar. So besteht die Gefahr, dass weiterhin viel Energie in die Arbeit an einem Beurteilungssystem investiert wird, das an den tatsächlichen Herausforderungen der Qualitätssicherung vorbeigeht.

\section{Qualitätstransparenz als Anreiz zur Qualitätsentwicklung}

Ungeachtet dieser Einwände ist unbestreitbar, dass die Transparenzkriterien in den Einrichtungen vieles in Bewegung gesetzt haben. Dies ist nicht auf die Kriterien selbst zurückzuführen, sondern schlicht darauf, dass Prüfergebnisse öffentlich wurden. Einrichtungen mit schlechten Noten hatten einen Rückgang der Nachfrage zu befürchten, was sich in vielen Fällen als ein stärkerer Ansporn erwies als die nichtöffentliche Forderung externer Prüfer, diesen oder jenen Qualitätsmangel zu beheben.

Die zugrunde liegende Idee einer öffentlichen Qualitätsberichterstattung mit vergleichenden Qualitätsbewertungen geht also in die richtige Richtung.
Gelänge es, inhaltlich-konzeptionelle und methodische Schwächen wie im aktuellen Verfahren zu vermeiden, könnte sie zu einem wichtigen Motor der Qualitätsentwicklung werden. Zwar wird immer wieder betont, dass Qualität nicht von außen in Pflegeeinrichtungen hineingeprüft werden könne, allerdings zeigt die Erfahrung, dass externe Prüfungen und Transparenz nach außen vielfach eine stärkere Motivation für die Qualitätsentwicklung erzeugen als der bloße Anspruch, Patienten und Bewohner zufrieden zu stellen und gute Pflege zu leisten.

Systeme der öffentlichen Qualitätsberichterstattung finden sich inzwischen in verschiedenen anderen Ländern. Sie sind meist angelegt als Informationsangebot für Nutzer oder potentielle Nutzer von Pflegeeinrichtungen. Pflegebedürftige bzw. ihre Angehörigen sollen damit in die Lage versetzt werden, bei der Auswahl einer Pflegeeinrichtung bzw. eines Pflegedienstes begründete Entscheidungen zu treffen. Das Internet ermöglicht einen bequemen Zugriff auf diese Informationen.

Das älteste System dieser Art findet sich in den USA (Mukamel et al. 2009). Dort kann anhand von bestimmten Kennziffern die Qualität einer bestimmten Einrichtung mit dem nationalen Durchschnitt oder dem Durchschnitt eines Bundesstaates verglichen werden. Ein ähnliches System gibt es in den Niederlanden (Steering Committee Responsible Care 2008). Großbritannien arbeitet weniger mit Kennzahlen als mit relativ ausführlichen Beschreibungen (öffentlichen Prüfberichten über die einzelnen Einrichtungen), die auf der Grundlage von externen Qualitätsprüfungen angefertigt werden und im Internet einsehbar sind. Abgesehen von einigen Ausnahmen steht die Entwicklung in den meisten Ländern jedoch noch am Anfang. Das Interesse an solchen Ansätzen ist groß, die mit ihnen verknüpften inhaltlichen und methodischen Herausforderungen sind allerdings nicht zu unterschätzen.

\section{Konzeptionelle Grundlagen der Qualitätsentwicklung}

Externe Prüfanforderungen in den Qualitätsrichtlinien von Kostenträgern sind ein wichtiger, aber keineswegs der einzige Bezugsrahmen für Qualitätssicherung und Qualitätsentwicklung in der Pflege. Seit der Anfangszeit der Pflegeversicherung haben die konzeptionellen Grundlagen der Pflege losgelöst von Prüfanforderungen erheblich an Umfang und Qualität gewonnen. Es existieren heute anspruchsvolle Standards, Rahmenkonzepte, Leistungsbeschreibungen und Interventionskonzepte, die Mitte der 1990er Jahre nicht einmal im Ansatz verfügbar waren.

Von besonderer Bedeutung sind in diesem Zusammenhang die nationalen Expertenstandards. Es gibt schon seit langem Standards für einzelne Pflegemaßnahmen, die beispielsweise in pflegerischen Ausbildungsgängen eingesetzt werden. Verbindliche Standards, die durch Forschungsergebnisse abgesichert sind und auf einem allgemeinen fachlichen Konsens beruhen, existieren jedoch erst seit verhältnismäßig kurzer Zeit: Seit etwas mehr als 10 Jahren werden in Deutschland nationale Expertenstandards für die Pflege entwickelt (Schiemann/Moers 2011), bislang zu den Arbeitsfeldern Dekubitusprophylaxe, Schmerzmanagement, Sturzprophylaxe, Förderung der Harnkontinenz, Pflege von Menschen mit chronischen Wunden und Ernährungsmanagement. Hinzu kommt ein Standard für pflegerisches Entlassungsmanagement im Krankenhaus.

Die Expertenstandards liefern eine wichtige Arbeitshilfe für die Praxis,

\section{Die Expertenstandards liefern eine wichtige Arbeitshilfe für die Praxis, stoßen gelegentlich jedoch auch auf Akzeptanzprobleme.}

stoßen gelegentlich jedoch auch auf Akzeptanzprobleme. Sie beanspruchen in der Regel für alle Aufgabenfelder der pflegerischen Versorgung Geltung, sind allerdings nicht auf alle Felder gleich gut zugeschnitten, was sie in der Umsetzung manchmal sperrig erscheinen lässt. Es kommt hinzu, dass sie zu einem wichtigen Bezugsrahmen bei externen Qualitätsprüfungen wurden und dadurch von einem Teil der Pflegenden weniger als Arbeitshilfe denn als professionsexterne Normen wahrgenommen werden. 
Das Pflege-Weiterentwicklungsgesetz hat die rechtliche Verbindlichkeit der Expertenstandards gestärkt und definierte außerdem ein Verfahren, in das zukünftige Standardentwicklungen einzubetten sind. Bislang liegen damit allerdings noch keine Erfahrungen vor. Der Übergang aus einer rein innerprofessionellen Initiative in einen Aushandlungsprozess mit Kostenträgern und anderen Beteiligten gestaltet sich offenbar schwierig.

Eine weitere wichtige Grundlage der Qualitätsentwicklung in der Pflege bilden Rabmenkonzepte für spezifische Aufgabenfelder. Dazu gehören im Heimbereich beispielsweise Rahmenkonzepte für die Angehörigenarbeit, die Begleitung Sterbender oder die Gestaltung des Überleitungsverfahrens bei Heimbewohnern, die in ein Krankenhaus aufgenommen werden müssen (vgl. KortePötters et al. 2007). Rahmenkonzepte definieren die inhaltlichen und formalen Anforderungen im jeweiligen Aufgabenfeld nur in allgemeiner Form und sind insofern mit den nationalen Expertenstandards nicht vergleichbar. Sie beziehen sich wie die angeführten Beispiele meist auf Arbeitsfelder, in denen ein standardisiertes Vorgehen nicht oder nur sehr begrenzt möglich ist. Rahmenkonzepte unterscheiden sich von den Expertenstandards häufig auch dadurch, dass sie nicht auf der Ebene der individuellen Pflege angesiedelt sind, sondern sich auf die Gestaltung von komplexeren Strukturen und Abläufen beziehen.

Leistungsbeschreibungen stellen einen weiteren wichtigen Bezugsrahmen zur Verfügung. Sie definieren, welche Leistungen von einer Einrichtung prinzipiell vorgehalten werden sollten, $d . h$. das angestrebte Leistungsprofil. Für die Qualitätsentwicklung und Qualitätsbeurteilung liefern Leistungsbeschreibungen ein Raster, mit dem unter anderem überprüft werden kann, ob die Leistungsfähigkeit der Einrichtung und die Qualifikation der Mitarbeiter dem Bedarf der jeweiligen Patienten-/ Bewohnergruppe entspricht. Rechtlich legitimierte Leistungsbeschreibungen existieren bislang nur in sehr allgemeiner Form in den Rahmenvereinbarungen zwischen Kostenträgern und Leistungserbringern. Konkrete, differenzierte Leistungsdefinitionen wurden für einzelne Bereiche der pflegerischen Versorgung in den letzten Jahren zwar ebenfalls entwickelt (vgl. Korte-Pötters et al. 2007), haben bisher aber keine Verbindlichkeit erlangt.

Anders als globale Leistungsbeschreibungen sind konkrete Interventionskonzepte auf bestimmte Bedarfskonstellationen bzw. auf bestimmte Personengruppen zugeschnitten. Beispiele hierfür sind Programme zur selbständigen Medikamenteneinnahme in der psychiatrischen Versorgung, die Unterstützung von Heimbewohnern mit Verhaltensproblemen, verschiedene Ansätze der Biografiearbeit bei kognitiv beeinträchtigten Personen oder die Unterstützung von Patienten mit somatischen Störungen im Umgang mit einer komplexen Medikation. In den letzten Jahren gab es in der Pflegewissenschaft verstärkte Bemühungen, solche und ähnliche Interventionskonzepte $\mathrm{zu}$ entwickeln und systematisch zu erproben (Schaeffer et al. 2008).

\section{Qualitätsmanagement}

Um die verfügbaren konzeptionellen Grundlagen wirksam werden zu lassen, ist ein funktionierendes internes Qualitätsmanagement (QM) unverzichtbar. Damit angesprochen sind die systematische Überwachung von Prozessen und Ergebnissen, die kontinuierliche Arbeit an den strukturellen Grundlagen (Personalressourcen, Qualifikation, Instrumente etc.) und die Durchführung planvoller Veränderungen im Versorgungsalltag.

Eine Zeit lang zogen komplexe QMSysteme wie das EFQM-Modell (European Foundation for Quality Management) oder die DIN EN ISO-Normen $9000 \mathrm{ff}$ in der Qualitätsdiskussion viel Aufmerksamkeit auf sich. Bislang sind unterschiedliche Erfahrungen mit solchen Ansätzen gemacht worden. Dazu gehört auch, dass komplexe QM-Systeme manchmal zu Formalismus tendieren und hohen bürokratischen Aufwand mit sich bringen. Für kleine Einrichtungen, beispielsweise für ambulante Pflegedienste, erweisen sich die Systeme zum Teil als zu kompliziert.

In engem Zusammenhang mit QMSystemen stehen Qualitätssiegel bzw. Zertifizierungen, über deren Nutzen die Meinungen auseinandergehen. So bezieht sich eine ISO-Zertifizierung lediglich auf die Beurteilung des QM selbst. Eine Einrichtung kann trotz eines Zertifikats eine schlechte Versorgungsqualität aufweisen. Für den Leistungsnutzer (oder den potenziellen Nutzer, der eine passende Einrichtung sucht) ist die begrenzte Aussagekraft einer Zertifizierung nicht ohne weiteres erkennbar. Ist ein Qualitätssiegel vorhanden, so die häufige Annahme, dann wird die Einrichtung auch eine gute Qualität vorweisen können (vgl. Geraedts/Selbmann, 2011).

Das interne Qualitätsmanagement vieler Pflegeeinrichtungen hat, wie eingangs erwähnt, seit Einführung der Pfle- 
wiesen, und auch eine öffentliche Qualitätsberichterstattung sollte auf verlässlichen, aussagekräftigen Kriterien aufbauen. In diesem Zusammenhang wird seit einigen Jahren verstärkt über den Einsatz von Ergebnisindikatoren diskutiert.

Bei externen Prüfungen erfolgt die Beurteilung von Ergebnisqualität bislang in Form von Einzelfallbeurteilungen und ohne Rückgriff auf Qualitätsindikatoren. Es wird also beurteilt, ob die gesundheitliche Situation und der »Pflegezustand « des Patienten/Bewohners dem entspricht, was man bei einer fachgerechten Versorgung erwarten darf, und ob durch fachliche Versäumnisse eine Gefährdung oder Schädigung eingetreten ist. Solche Beurteilungen sind auch im internen Qualitätsmanagement durchführbar, etwa im Rahmen von Pflegevisiten. Für eine vergleichende Qualitätsbewertung, also den Vergleich der Ergebnisqualität verschiedener Einrichtungen, böte dies allerdings keine geeignete Grundlage.

Inzwischen liegt ein neuer, praktisch erprobter Ansatz vor, der zumindest in der vollstationären Langzeitpflege vergleichende, indaktorengestützte Qualitätsbeurteilungen auf der Ebene der Ergebnisqualität ermöglicht. Er ist das Ergebnis eines Entwicklungsprojekts, das vom Bundesministerium für Gesundheit und dem Bundesministerium für $\mathrm{Fa}$ milie, Senioren, Frauen und Jugend in Auftrag gegeben wurde (Wingenfeld et al. 2011).

Pflegeergebnisse sind - darin besteht in der internationalen Diskussion weitgehende Einigkeit - Veränderungen des Gesundheitszustands, des Verhaltens und des Erlebens von Pflegebedürftigen, die (vorrangig) auf pflegerische Maßnahmen zurückzuführen sind (vgl. Arling et al. 2005). Der Begriff Veränderung ist hierbei weit zu interpretieren. Der Erhalt der Status Quo (z. B. der Erhalt der Mobilität), der bei schwer kranken Menschen ein vorrangiges Versorgungsziel darstellen kann, ist ebenfalls als Ergebnis pflegerischer Versorgung zu verstehen.

Indikatoren für Ergebnisqualität müssen zahlreichen methodischen Anforderungen genügen, wenn mit ihrer Hilfe vergleichende Qualitätsbeurteilungen erfolgen sollen. Die während des Projekts entwickelten Qualitätsindika- toren und Instrumente wurden während einer zehnmonatigen Praxisphase in 46 vollstationären Pflegeeinrichtungen getestet. Jene Indikatoren, die aufgrund dieser Erfahrung als geeignet für einen Qualitätsvergleich befunden wurden, gliedern sich in fünf Bereiche (jeder der Bereiche umfasst mehrere Indikatoren):

1. Erhalt und Förderung der Selbständigkeit (z. B. Selbständigkeit bei Alltagsverrichtungen)

2. Schutz vor gesundheitlichen Schädigungen und Belastungen (Dekubitusentstehung, Gewichtsabnahme, Stürze mit gravierenden Folgen)

3. Unterstützung bei spezifischen Bedarfslagen (u. a. mit den Themen Schmerzmanagement und herausforderndes Verhalten)

4. Wohnen und (hauswirtschaftliche) Versorgung (z. B. Sauberkeit, Beurteilung der Mahlzeiten)

5. Tagesgestaltung und soziale Beziehung (z. B. Beurteilung des Angebots an Gruppenaktivitäten oder Respektieren der Privatsphäre).

\section{Indikatoren für Ergebnis-} qualität müssen zahlreichen methodischen Anforderungen genügen, wenn mit ihrer Hilfe vergleichende Qualitätsbeurteilungen erfolgen sollen.

Die Indikatoren in den Bereichen 4 und 5 werden vorrangig über eine Bewohnerund Angehörigenbefragung erfasst. Alle übrigen erfordern Stichtagserhebungen im Abstand von sechs Monaten, bei denen eine Einschätzung grundlegender Fähigkeiten des Bewohners erfolgt und ausgewählte Informationen aus der vorhandenen Dokumentation extrahiert werden.

Charakteristisch für die Erfassung von Ergebnisqualität ist, dass anders als im Falle der Prozessqualität nicht versucht wird, Entscheidungen und Handlungen der Pflegenden zu rekonstruieren. Vielmehr konzentriert sich der Blick auf die Situation des Bewohners und seine Bewertung des Leistungsgeschehens. So wird beispielsweise der
Anteil der Bewohner ermittelt, bei denen sich die Mobilität innerhalb eines Zeitraumes von sechs Monaten nicht verschlechtert (oder verbessert) hat. Das Ergebnis wird für Bewohner, die erhebliche bis schwerste kognitive Einbußen aufweisen, getrennt vom Ergebnis für die übrigen Bewohner dargestellt, denn die Chancen des Mobilitätserhalts sind je nach kognitiven Einbußen sehr unterschiedlich. Ein anderer Indikator erfasst den Anteil der Bewohner mit hohem Dekubitusrisiko, die innerhalb der letzten sechs Monate in der Einrichtung (nicht im Krankenhaus oder einer anderen Umgebung) ein Druckgeschwür 2. bis 4 . Grades entwickelt haben. Auch hier erfolgt eine Gruppenunterscheidung, weil die Häufigkeit der Dekubitusentstehung erheblich von der Eigenbeweglichkeit (Mobilität) der Bewohner abhängt. In ähnlicher Weise werden Beurteilungen der Bewohner abgebildet, beispielsweise als Anteil der Bewohner, die sich von den Mitarbeitern respektvoll behandelt fühlen.

\section{Ein neues Zusammenspiel von internem Qualitätsmanagement und externen Prüfungen?}

Im Hinblick auf einen möglichen Einsatz von Ergebnisindikatoren ergeben sich je nach Indikatorengruppe spezifische Anforderungen. Die Bewohnerbefragung erfordert organisatorische Lösungen, die unerwünschte Einflüsse auf die Antworten der Bewohner ausschließen. Die Befragung muss daher durch eine neutrale externe Stelle bzw. Person durchgeführt werden.

Die im Projekt entwickelten Indikatoren der Bereiche 1 bis 3 hingegen legen eine neue Form des Zusammenspiels zwischen externen Prüfungen und internem Qualitätsmanagement der Einrichtungen nahe. Aus methodischen Gründen kann bei der indikatorengestützten Beurteilung von Ergebnisqualität nicht mit kleinen Stichproben gearbeitet werden, vielmehr ist je Einrichtung die Gesamtheit der Bewohner einzubeziehen. Dies wiederum ist nur mit Hilfe von Daten möglich, die von den Einrichtungen selbst bereitgestellt werden. Der im Projekt entwickelte Ansatz sieht daher perspektivisch folgende Arbeitsteilung vor: 
- Im Rahmen des internen Qualitätsmanagements erfolgt in regelmäßigen Abständen (z. B. alle sechs Monate) eine Datenerhebung (Zusammenstellung von Daten aus der Routinedokumentation und Einschätzung von ausgewählten Aspekten der Pflegebedürftigkeit).

- Eine reine Selbstbewertung der Einrichtungen wäre jedoch kritisch zu hinterfragen, deshalb wird eine externe Kontrolle der Verlässlichkeit der Datenerfassungen in den Einrichtungen durch externe Prüfungen vorgeschlagen. Externe Prüfer hätten also die Richtigkeit der Daten stichprobenartig zu kontrollieren. Hierzu würden Stichprobengrößen, wie sie für die heutigen MDK-Prüfungen festgelegt sind, ausreichen.

Für das interne Qualitätsmanagement würde ein solcher Ansatz einen überschaubaren Mehraufwand mit sich bringen. Auch für externe Prüfungen ergäben sich eher moderate Änderungen, denn viele Informationen, die zur Erfassung von Ergebnisqualität benötigt werden, sind bereits heute ein Thema bei den externen Prüfungen. Nicht zu unterschätzen sind allerdings die fachlichen und logistischen Anforderungen eines solchen Systems. Der Umgang mit standardisierten Einschätzungsinstrumenten ist in vielen Einrichtungen noch keine Routine. Auch die Entwicklung einer Infrastruktur, mit der große Datenmengen verwaltet werden können, wäre eine Herausforderung.

Mit dem im Projekt entwickelten Ansatz könnte der Anspruch, Ergebnisqualität in den Mittelpunkt von Qualitätssicherung und Qualitätsbeurteilung zu stellen, nach vielen Jahren Diskussion umgesetzt werden. Der für die Bewohner wichtigste Effekt wäre eine Stärkung und Verbesserung des internen Qualitätsmanagements, das für eine nachhaltige Sicherung von Qualität von elementarer Bedeutung ist. Die Eigenverantwortung der Einrichtungen würde erheblich gestärkt und ein starker Anreiz für "gute Pflege « im Interesse des Bewohners geschaffen.

\section{Ausblick}

Die im vorliegenden Beitrag skizzierten Fragen der Qualitätssicherung und Qualitätsentwicklung umfassen nicht sämt- liche Herausforderungen, die sich heute auf dem Feld der pflegerischen Versorgung stellen. Unberücksichtigt blieb beispielsweise die Parallelität der externen Prüfsysteme (Medizinische Dienste und Heimaufsicht), deren Abstimmung nach der Föderalismusreform, d. h. nach dem Übergang des Heimrechts in die Verantwortung des jeweiligen Bundeslandes, noch schwieriger geworden ist. Auch die wichtige Frage nach den personellen Voraussetzungen für die Sicherung und Weiterentwicklung von Qualität blieb ausgespart.

Deutlich geworden ist jedoch hoffentlich, dass heute, mehr als 15 Jahre nach der Einführung der Pflegeversicherung, Optionen einer grundlegenden Reorganisation der herkömmlichen Strukturen zur Qualitätssicherung in der Pflege in den Blick genommen werden müssen. Es hat viele Entwicklungen und Fortschritte in dieser Zeit gegeben, aber auch viel Erkenntnis- und Erfahrungsgewinn, der auf die Grenzen der bisherigen Lösungen verweist.

Zukünftige Formen der Beurteilung und Weiterentwicklung von Qualität werden stärker die Ergebnisqualität in den Mittelpunkt stellen. In diesem Zusammenhang, aber auch aus anderen Gründen ist die künftige Arbeitsteilung zwischen internem Qualitätsmanagement und externen Prüfdiensten zu diskutieren. Die Erfahrungen mit externen Prüfungen nach der Einführung der Transparenzkriterien werfen inhaltliche und methodische Grundsatzprobleme auf, die nicht hinter der pragmatischen Diskussion um Detailfragen zurückstehen sollten. Es müssen auch effektivere Wege gefunden werden, die verbesserten inhaltlich-konzeptionellen Grundlagen der pflegerischen Versorgung in der Praxis stärker zu verankern. Auch die Frage nach der Anpassung von Qualitätssicherungsansätzen an den jeweiligen Versorgungsbereich erfordert mehr Aufmerksamkeit.

Kurz: Die aktuellen Bemühungen zur Modernisierung der pflegerischen Versorgung, die angesichts der demografischen Herausforderungen immer dringlicher wird, müssen sich auch auf neue und effektive Formen der Qualitätssicherung richten.

\section{Literatur}

Arling, G./Kane, R.L./Lewis, T./Mueller, C. (2005): Future development of nursing home quality indicators. The Gerontologist 45 , Nr. 2, 147-156

Geraedts, M., Selbmann, H.-K. (2011): Konzepte des Qualitätsmanagements. In: Schaeffer, D., Wingenfeld, K. (Hrsg.): Handbuch Pflegewissenschaft (Neuausgabe). Juventa, Weinheim/München, 599-616

Körte-Potters, U., Wingenfeld, K., Heitmann, D. (2007): Konzepte zur Sicherstellung der Versorgungsqualität in vollstationären Einrichtungen. In: Ministerium für Arbeit Gesundheit und Soziales des Landes Nordrhein-Westfalen (MAGS) (Hrsg.): Referenzmodelle. Band 5: Oualitätsverbesserung in der vollstationären Pflege - Leitfaden zur praktischen Umsetzung des Referenzkonzepts. Düsseldorf: MAGS

Mukamel, D.B./Ladd, H./Weimer, D.L./ Spector, W.D./Zinn, J.S. (2009): Is There Evidence of Cream Skimming Among Nursing Homes Following the Publication of the Nursing Home Compare Report Card? Gerontologist 49, Nr. 6, 793-802

Newsletter Pflegenoten 2011: Aktuelle Statistiken aus der DCS - Pflege. http://www. vdek.com/vertragspartner/Pflegeversicherung/Newsletter_Pflegenoten/dcs_monatliche_statistik_20111205.pdf

Schaeffer, D./Behrens, J./ Görres, S. (Hrsg.) (2008): Optimierung und Evidenzbasierung pflegerischen Handelns. Ergebnisse und Herausforderungen der Pflegeforschung. Juventa, Weinheim/München

Schiemann, D./Moers, M. (2011): Qualitäts entwicklung und -standards in der Pflege. In: Schaeffer, D., Wingenfeld, K. (Hrsg.): Handbuch Pflegewissenschaft (Neuausgabe). Juventa, Weinheim/München, 617-642

Steering Committee Responsible Care (2008): Quality Framework Responsible Care. Rijswijk

Wingenfeld, K./Kleina, T./Franz, S./Engels, D./Mehlan, S./Engel, H. (2011): Entwicklung und Erprobung von Instrumenten zur Beurteilung der Ergebnisqualität in der stationären Altenhilfe. Hg. Vom Bundesministerium für Gesundheit und vom Bundesministerium für Familie, Senioren, Frauen und Gesundheit. Bielefeld/Köln (http://www.bmg. bund.de/fileadmin/dateien/Downloads/P/ Pflegeheim/Abschlussbericht_Ergebnisqualitaet_stationaere_Altenhilfe_20110601.pdf v. 20. Dezember 2011) 